\title{
Características Fisiológicas das Culturas de Soja e FeiJão e de Três Espécies de Plantas Daninhas ${ }^{1}$
}

\author{
Physiological Caracteristics of Soybean and Common Bean Crops and Three Weed Species
}

PROCÓPIO, S.O. ${ }^{2}$, SANTOS, J.B. ${ }^{3}$, SILVA, A.A. ${ }^{4}$, MARTINEZ, C.A. ${ }^{5}$ e WERLANG, R.C. ${ }^{3}$

\begin{abstract}
RESUMO - O objetivo do trabalho foi avaliar os características fisiológicas taxa de fotossíntese líquida (A), taxa de transpiração (E), condutância estomática (gs) e eficiência do uso da água (WUE) e os índices de crescimento biomassa seca total (Wt) e índice de área foliar (L) para as culturas de soja (Glycine max) e feijão (Phaseolus vulgaris) e para as das plantas daninhas Bidens pilosa, Desmodium tortuosum e dois biótipos de Euphorbia heterophylla (um suscetivel e outro resistente aos herbicidas inibidores da ALS), em duas épocas de avaliação: 39 e 67 dias após a semeadura (DAS). O experimento foi conduzido a campo em um Argissolo Vermelho-Amarelo, fase terraço, utilizando-se o delineamento experimental de blocos ao acaso, com parcelas subdivididas, sendo o fator da parcela principal as espécies vegetais e o da subparcela as épocas de avaliação, com quatro repetições por tratamento. Foi observado maior Wt da soja em relação às plantas daninhas. As culturas de soja e feijão apresentaram maior L do que as plantas daninhas. Os biótipos de E. heterophylla apresentaram a maior A, bem como a maior gs. Com exceção de $D$. tortuosum, as plantas daninhas apresentaram maior WUE em relação às culturas nos estádios iniciais de desenvolvimento. Não foram observadas diferenças em relação a qualquer parâmetro fisiológico avaliado entre os biótipos de E. heterophylla.
\end{abstract}

Palavras-chave: Phaseolus vulgaris, Glycine max, fotossíntese, transpiração.

\begin{abstract}
This work aimed to evaluate the physiological caracteristics net photosynthesis (A), transpiration rate (E), stomatal conductance (gs) and water use efficiency (WUE) and growth indexes of total dry matter (Wt) and leaf area index (L) for soybean (Glycine max), common bean (Phaseolus vulgaris) and weeds Bidens pilosa, Desmodium tortuosum and two biotypes of Euphorbia heterophylla (one susceptible and other resistant to ALS inhibitor herbicides) during two periods of evaluation, 39 and 67 days after sowing (DAS). The experiment was conducted under field conditions in a Red-yellow Clayoil, in a randomized complete block arrangement, with four replications, in a split-plot design. The species were the plots and the evaluation times the split-plots. Higher Wt of soybean was observed in relation to the weeds. The soybean and common bean crops showed higher $L$ than the weeds. E. heterophylla biotypes presented the largest $A$ and gs values. Except for $\boldsymbol{D}$. tortuosum, the weeds presented larger WUE at the initial stage of crops development. No differences were observed between E. heterophylla biotypes.
\end{abstract}

Key words: Phaseolus vulgaris, Glycine max, photosynthesis, transpiration.

Recebido para publicação em 10.4.2003 e na forma revisada em 5.3.2004.

2 Prof. do Dep. de Fitossanidade da Universidade Federal de Pelotas - UFPEL, 96010-900 Pelotas-RS. ${ }^{3}$ Doutorando, Dep. de Fitotecnia da Universidade Federal de Viçosa - UFV, <jbarbosasantos@yahoo.com.br>; ${ }^{4}$ Prof. Dep. de Fitotecnia - UFV, <aasilva@ufv.br>; ${ }^{5}$ Prof. Dep. de Biologia, FFCLRP, USP, Ribeirão Preto-SP, <carlosamh@ffclrp.usp.br>; ${ }^{3}$ Doutorando, Dep. de Fitotecnia - UFV, <werlang@ hotmail.com>. 


\section{INTRODUÇÃO}

O aumento na contaminação do meio ambiente por pesticidas vem preocupando várias nações, principalmente as que apresentam atividades agrícolas intensas, com grande utilização desses insumos. Os herbicidas representam a fração dos pesticidas com maior volume de aplicação nos sistemas de produção vegetal. A cada safra, os herbicidas vêm se tornando a principal ferramenta utilizada no manejo integrado das plantas daninhas (MIPD). O custo, o rendimento operacional e a alta seletividade destes produtos às culturas são tidos como as principais razões para o aumento da sua utilização. Outros métodos de controle, como o controle cultural, também podem contribuir efetivamente para o MIPD. Contudo, para se extrair o máximo proveito do controle cultural, proporcionando vantagens competitivas às culturas diante das plantas daninhas, é necessário conhecer a fisiologia destas espécies.

Práticas culturais como a escolha do cultivar, a determinação do espaçamento e a profundidade de semeadura, o volume e a época de irrigação, a quantidade e a localização da adubação e a época de semeadura são estratégias que podem auxiliar no MIPD, desde que sejam conhecidos os pontos fortes e fracos da "nossa equipe" (as culturas agrícolas) e dos "nossos adversários momentâneos" (as plantas daninhas). Begna et al. (2001) relatam que a simples troca de um híbrido de milho por outro com características morfológicas mais competitivas pode resultar na redução dos niveis necessários de herbicidas para o controle local das plantas daninhas.

A maioria dos estudos em que se avaliaram os efeitos da competição entre as plantas daninhas e as culturas (Vieira, 1970; Blanco et al., 1973; Coble et al., 1981; Mascarenhas, 1982; Vargas et al., 1999; Vidal \& Trezzi, 2000; Santos $\&$ Silva, 2000) tiveram como objetivo apenas avaliar o efeito dessas plantas na produtividade e/ou no crescimento (acúmulo de biomassa) das culturas, ou seja, quantificar as conseqüências da presença das plantas daninhas, sem estudar as causas relacionadas às aptidões fisiológicas específicas de cada planta.

Biótipos de Euphorbia heterophylla, resistentes aos herbicidas inibidores da ALS, foram identificados em lavouras de soja nos
Estados do Rio Grande do Sul, Paraná e Mato Grosso do Sul, onde estes produtos são empregados há mais de seis anos (Vargas et al., 1999). Alguns trabalhos mostraram não haver diferença competitiva entre os biótipos sensiveis e resistentes de E. heterophylla quanto a morfologia, duração do ciclo e potencial de competição com a cultura da soja (Vargas et al., 1999; Vidal \& Trezzi, 2000; Santos \& Silva, 2000). Masabni \& Zandstra (2000), avaliando as características fisiológicas de dois biótipos de Portulaca oleracea (um suscetivel e outro resistente ao herbicida linuron), observaram que a $30{ }^{\circ} \mathrm{C}$ a taxa de assimilação de $\mathrm{CO}_{2}$ do biótipo suscetivel era maior, mas não diferia do biótipo resistente a $40{ }^{\circ} \mathrm{C}$. Dominguez et al. (1994) verificaram que um biótipo de Solanum nigrum, suscetivel a herbicidas do grupo das triazinas, apresentou maior crescimento do que um biótipo resistente a este grupo de herbicidas, resultado que corrobora o encontrado por Kremer \& Kropff (1999).

O objetivo deste trabalho foi avaliar características fisiológicas das culturas de soja (Glycine max) e feijão (Phaseolus vulgaris) e das plantas daninhas Bidens pilosa, Desmodium tortuosum e de dois biótipos de Euphorbia heterophylla (um suscetivel e outro resistente aos herbicidas inibidores da ALS).

\section{MATERIAL E MÉTODOS}

O experimento foi conduzido em campo experimental da Universidade Federal de Viçosa (UFV), no período de outubro de 2001 a fevereiro de 2002, em solo classificado como Argissolo Vermelho-Amarelo, fase terraço.

Os tratamentos - num total de 12 - foram compostos pelas culturas de soja (cultivar UFV19) e feijão (cultivar Pérola), pelas espécies de plantas daninhas Euphorbia heterophylla (suscetivel a inibidores de ALS), Euphorbia heterophylla (resistente a inibidores de ALS), Bidens pilosa e Desmodium tortuosum e por duas épocas de avaliação: 39 e 67 dias após a semeadura (DAS). O delineamento experimental utilizado foi o de blocos ao acaso com parcelas subdivididas, sendo o fator da parcela principal as espécies vegetais e o da subparcela as épocas de avaliação. Foram utilizadas quatro repetições por tratamento. A subparcela constou de dez metros de largura por dez de 
comprimento, contendo 16 fileiras da espécie vegetal avaliada.

O sistema de preparo do solo adotado na área experimental foi o convencional, com uma aração e duas gradagens. A adubação de plantio foi realizada de maneira uniforme em toda a área experimental, utilizando-se $500 \mathrm{~kg}$ da fórmula 4-14-8 por hectare. Foi realizada escarificação nas sementes de $D$. tortuosum, com a finalidade de quebra de dormência. A semeadura foi manual. Após a emergência das plantas (aproximadamente seis dias após a semeadura), realizou-se desbaste, deixandose cinco plantas por metro em todos os tratamentos. Todas as parcelas foram mantidas livres da presença de outras plantas por meio de capinas manuais.

A taxa de fotossintese líquida (A), a taxa de transpiração $(\mathbf{E})$ e a condutância estomática (gs) foram avaliadas na parte mediana de folhas ou foliolos, completamente expandidos, presentes no terço superior das plantas. Foi utilizado um analisador de gases no infravermelho (IRGA), marca ADC, modelo LCA 2 (Analytical Development Co. Ltd, Hoddesdon, UK) em sistema aberto. As avaliações foram realizadas entre 8 e 10 horas da manhã, escolhendo-se três plantas ao acaso por parcela. A área foliar das plantas foi determinada por meio de um medidor eletrônico de área foliar (Delta T. Devices), e a biomassa seca total (Wt) das plantas foi obtida por pesagem em balança de precisão após a secagem destas em estufa a $75^{\circ} \mathrm{C}$ por 72 horas. O indice de área foliar (L) foi calculado dividindo-se a área foliar das plantas em $\mathrm{m}^{2}$ pela superfície do terreno, também em $\mathrm{m}^{2}$. A eficiência do uso da água (WUE) foi calculada mediante a relação entre a quantidade de $\mathrm{CO}_{2}$ fixado através da fotossíntese e a quantidade de água transpirada (Nobel, 1991).

Os dados meteorológicos diários foram obtidos da Estação Meteorológica do Departamento de Engenharia Agrícola da UFV, instalada a aproximadamente $400 \mathrm{~m}$ da área experimental, sendo apresentados nas Figuras 1,2 e 3.

Após coleta e tabulação, os dados foram submetidos à análise de variância e as médias comparadas pelo teste de Tukey a $5 \%$ de probabilidade.

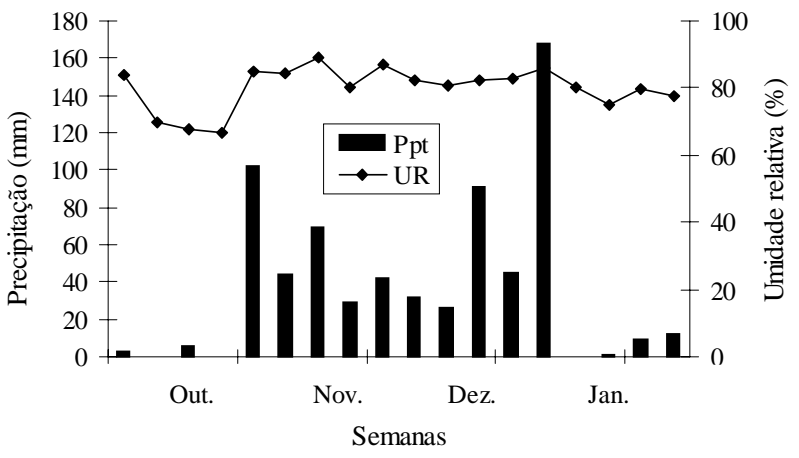

Figura 1 - Médias semanais de precipitação (Ppt) e umidade relativa (UR), no período de outubro de 2000 a fevereiro de 2001. Viçosa-MG.

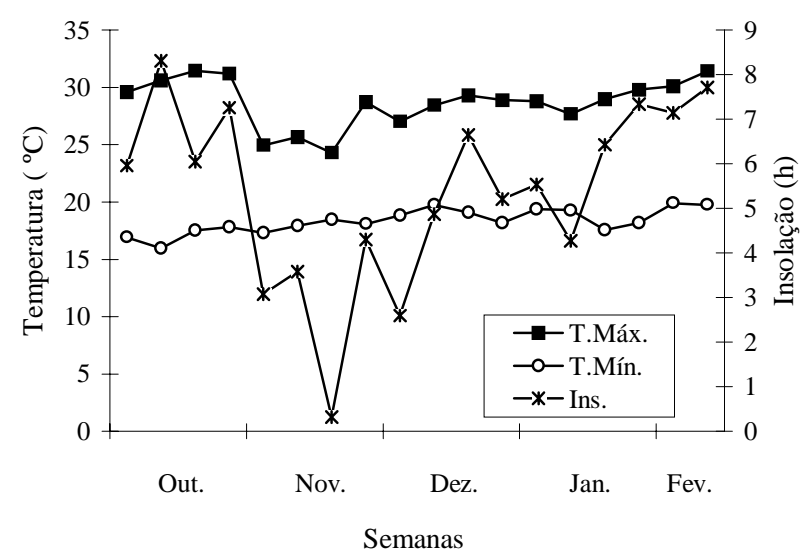

Figura 2 - Médias semanais de temperatura máxima (T. Máx), temperatura mínima (T. Mín.) e insolação (Ins.), no período de outubro de 2000 a fevereiro de 2001. Viçosa-MG.

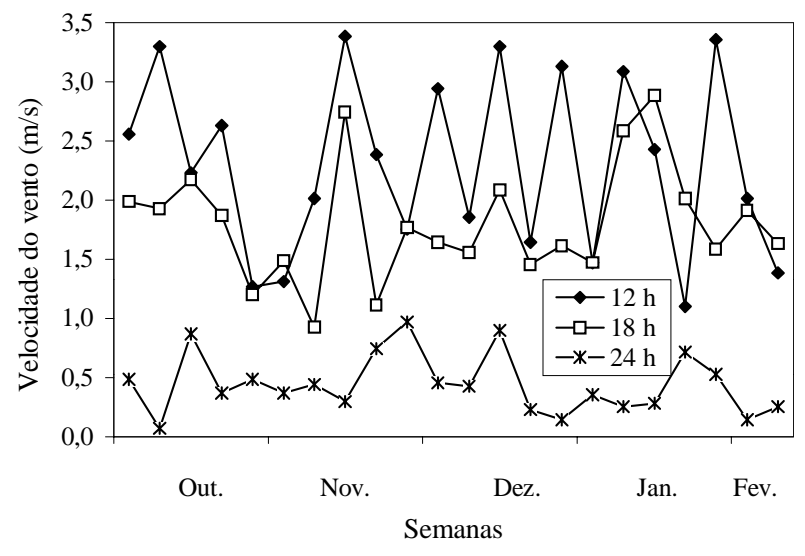

Figura 3 - Médias semanais de velocidade do vento às 12 horas (12 h), 18 horas (18 h) e às 24 horas ( $24 \mathrm{~h})$, no período de outubro de 2000 a fevereiro de 2001. ViçosaMG. 


\section{RESULTADOS E DISCUSSÃO}

Aos 39 DAS, o feijão apresentou a maior biomassa seca total (Wt) entre as espécies avaliadas (Tabela 1). A emergência mais rápida (até cinco dias antes das outras espécies) e a maior taxa de acúmulo de biomassa seca na fase vegetativa podem explicar o maior valor de Wt apresentado pelas plantas de feijão. Foi observado maior Wt da soja em relação às das plantas daninhas, tanto aos 39 como aos 67 DAS (Tabela 1). Entre as plantas daninhas, os biótipos de $E$. heterophylla não diferiram em relação a Wt, em ambos os períodos avaliados, e aos 39 DAS apresentaram maior Wt do que $B$. pilosa e D. tortuosum. Esta última foi a espécie que acumulou a menor Wt aos 67 DAS, sendo uma espécie de crescimento inicial muito lento e de ciclo longo (137 dias), características que exprimem baixo potencial competitivo. Todas as espécies tiveram aumento significativo de Wt entre os períodos avaliados (Tabela 1).

As culturas de soja e feijão apresentaram maior índice de área foliar (L) do que as plantas daninhas, tanto aos 39 quanto aos 67 DAS (Tabela 1). Todavia, aos 67 DAS, o L da soja foi maior que o do feijão. Entre as plantas daninhas, aos 39 DAS, não houve diferença quanto ao $\mathbf{L}$ entre todas as espécies avaliadas. Já aos 67 DAS o maior $\mathbf{L}$ foi observado em plantas de D. tortuosum (Tabela 1). O L dos biótipos de E. heterophylla não diferiu nos dois periodos avaliados. Sterling et al. (2001) não encontraram diferenças na habilidade competitiva de dois biótipos de Centaurea solstitialis, um suscetivel e outro resistente ao herbicida picloram. Dos 39 para os 67 DAS foi detectado aumento significativo do $\mathbf{L} \mathrm{em}$ todas as espécies (Tabela 1). Como o $\mathbf{L}$ é um das caracterísitcas que podem indicar a capacidade das plantas em competir por luz, percebe-se que as culturas da soja e do feijão apresentaram maior potencial de competição por esse recurso do que as plantas daninhas.

Os biótipos de E. heterophylla apresentaram a maior taxa de fotossintese líquida por unidade de área foliar (A) aos 39 e aos 67 DAS, não havendo diferença entre eles; contudo, nesta última data, não diferiram das plantas de soja e de B. pilosa (Tabela 1). Sharples et al. (1997) constataram que a taxa fotossintética líquida, sob fluxo de fótons de $350 \mu \mathrm{mol} \mathrm{m} \mathrm{m}^{-2} \mathrm{~s}^{-1}$, não diferiu entre um biótipo de Alopecurus myosurus suscetivel e outro resistente ao herbicida chlorotoluron. Aos 39 DAS, D. tortuosum apresentou a menor $\mathbf{A}$ entre as espécies avaliadas, fato este que também ajuda a explicar seu baixo desempenho em acumular biomassa no período. As plantas de feijão apresentaram a menor $\mathbf{A}$ aos 67 DAS, período no qual as plantas estavam formando grãos, diferentemente das demais, que estavam no florescimento (soja, B. pilosa e biótipos de $E$. heterophylla) ou ainda na fase vegetativa ( $D$. tortuosum). A A diminuiu da primeira para a segunda avaliação nas plantas de feijão e nos biótipos de E. heterophylla, não se alterou nas plantas de soja e de B. pilosa e aumentou nas plantas de D. tortuosum. Esse

Tabela 1 - Biomassa seca total (Wt), índice de área foliar (L) e taxa de fotossíntese líquida (A), observados nas culturas de soja e feijão e em plantas daninhas aos 39 e 67 DAS. Viçosa-MG, 2001

\begin{tabular}{|c|c|c|c|c|c|c|}
\hline \multirow[t]{2}{*}{ Tratamento } & \multicolumn{2}{|c|}{$\mathbf{W t}(\mathrm{g})$} & \multicolumn{2}{|c|}{$\mathbf{L}$} & \multicolumn{2}{|c|}{$\begin{array}{c}\mathbf{A} \\
\left(\mu \mathrm{mol} \mathrm{CO} \mathrm{m}^{-2} \mathrm{~s}^{-1}\right)\end{array}$} \\
\hline & 39 DAS & 67 DAS & 39 DAS & 67 DAS & 39 DAS & 67 DAS \\
\hline P. vulgaris & 3,626 a $\mathrm{B}$ & 19,900 a $\mathrm{A}$ & 0,344 a B & $0,652 \mathrm{~b} \mathrm{~A}$ & $11,850 \mathrm{c} \mathrm{A}$ & 8,742 c B \\
\hline G. $\max$ & $2,185 \mathrm{~b} \mathrm{~B}$ & 24,485 a $\mathrm{A}$ & 0,302 a B & 0,815 a $\mathrm{A}$ & $12,442 \mathrm{bc} \mathrm{A}$ & $12,825 \mathrm{ab} \mathrm{A}$ \\
\hline E. heterophylla (resistente*) & 0,682 c B & $13,062 \mathrm{~b} \mathrm{~A}$ & 0,115 b B & $0,305 \mathrm{~cd} \mathrm{~A}$ & 16,475 a $\mathrm{A}$ & 14,400 a B \\
\hline E. heterophylla (suscetível*) & 0,689 c B & $12,353 \mathrm{~b} \mathrm{~A}$ & $0,125 \mathrm{~b} \mathrm{~B}$ & $0,230 \mathrm{~d} \mathrm{~A}$ & 17,341 a $A$ & 14,566 a B \\
\hline B. pilosa & $0,300 \mathrm{~d} \mathrm{~B}$ & $14,322 \mathrm{~b} \mathrm{~A}$ & $0,203 \mathrm{~b} \mathrm{~B}$ & 0,436 c A & $13,867 \mathrm{~b} \mathrm{~A}$ & $12,709 \mathrm{ab} \mathrm{A}$ \\
\hline D. tortuosum & $0,245 \mathrm{~d} \mathrm{~B}$ & $4,692 \mathrm{c} \mathrm{A}$ & $0,101 \mathrm{~b} \mathrm{~B}$ & $0,598 \mathrm{~b} \mathrm{~A}$ & $8,950 \mathrm{~d} \mathrm{~B}$ & $11,450 \mathrm{~b} \mathrm{~A}$ \\
\hline
\end{tabular}

* Biótipo resistente e suscetível aos herbicidas inibidores da ALS.

Médias seguidas de mesma letra, minúscula na coluna e maiúscula na linha, não diferem estatisticamente entre si pelo teste de Tukey a $5 \%$ de probabilidade. 
fato demonstra uma tendência de correlação da $\mathbf{A}$ com o ciclo das espécies: plantas mais precoces, queda na $\mathbf{A}$; plantas mais tardias, aumento na $\mathbf{A}$.

Mesmo os biótipos de E. heterophylla que apresentaram maior $\mathbf{A}$ do que as plantas de soja aos 39 DAS tiveram a sua área foliar menor nesse periodo, sendo provavelmente uma das razões que fizeram com que a Wt das plantas de soja fosse, aproximadamente, três vezes maior que a dos biótipos de E. heterophylla.

Os biótipos de E. heterophylla apresentaram maior condutância estomática (gs) aos 39 DAS (Tabela 2), fato este que se correlaciona com a maior $\mathbf{A}$ apresentada nesta data, possivelmente por permitir maior fluxo de entrada de $\mathrm{CO}_{2}$. A gs, aos 67 DAS, não diferiu entre as espécies avaliadas. Foi detectado aumento neste parâmetro para todas as plantas avaliadas entre 39 e 67 DAS (Tabela 2), ou seja, uma diminuição na resistência estomática, possivelmente devido à maior umidade relativa do ar (UR) observada na segunda data de avaliação.

Aos 39 DAS, constatou-se que a taxa de transpiração por unidade de área foliar (E) não diferiu entre as espécies avaliadas (Tabela 2). Já aos 67 DAS observou-se que o biótipo de $E$. heterophylla suscetivel aos inibidores da ALS apresentou maior $\mathbf{E}$ do que as culturas de soja e feijão, porém não diferindo do biótipo resistente. Em todas as espécies verificou-se diminuição da $\mathbf{E}$ em relação aos 39 para os 67 DAS (Tabela 2). Também, como já relatado para a gs, a maior UR observada na segunda avaliação deve ser o motivo da diminuição da E.

Aos 39 DAS, os biótipos de E. heterophylla apresentaram a melhor eficiência no uso da água (WUE), porém não diferindo de $B$. pilosa (Tabela 2). Nesse periodo, D. tortuosum foi a planta com menor WUE. Com exceção desta espécie, as outras plantas daninhas apresentaram maior WUE em relação às culturas. Aos 67 DAS, os biótipos de E. heterophylla, soja e $B$. pilosa foram as espécies que apresentaram a melhor WUE. Também nesta época constatou-se que as plantas de soja apresentaram maior WUE do que as de feijão. Pela Tabela 2, observa-se aumento na WUE, entre os períodos avaliados, nas plantas de soja e $D$. tortuosum, que foram as espécies mais tardias; diminuição nas plantas de feijão (a espécie mais precoce); e não-alteração nos biótipos de $E$. heterophylla e B. pilosa, espécies de ciclo intermediário em relação às demais. A alta eficiência no uso da água é característica presente em plantas voltadas à sobrevivência e perpetuação, que sobrevivem em ambientes limitados em recursos naturais, como a grande parte das plantas daninhas.

Pode-se concluir, em termos gerais, que as plantas daninhas produziram menor biomassa seca e apresentaram menor enfolhamento do que as culturas; no entanto, elas foram mais eficientes em aproveitar o recurso luz por unidade de área foliar, bem como em utilizar melhor (maior economia) a água absorvida, com

Tabela 2 - Condutância estomática (gs), taxa de transpiração (E) e eficiência no uso da água (WUE), observadas nas culturas de soja e feijão e em plantas daninhas aos 39 e 67 DAS. Viçosa-MG, 2001

\begin{tabular}{|c|c|c|c|c|c|c|}
\hline \multirow[t]{2}{*}{ Tratamento } & \multicolumn{2}{|c|}{$\begin{array}{c}\mathbf{g s} \\
\left(\mathrm{mol} \mathrm{m}^{-2} \mathrm{~s}^{-1}\right)\end{array}$} & \multicolumn{2}{|c|}{$\begin{array}{c}\mathbf{E} \\
\left(\mathrm{mmol} \mathrm{H} \mathrm{O} \mathrm{m}^{-2} \mathrm{~s}^{-1}\right) \\
\end{array}$} & \multicolumn{2}{|c|}{$\begin{array}{c}\text { WUE } \\
\left(\mu \mathrm{mol} \mathrm{CO}_{2} \mathrm{mmol} \mathrm{H}_{2} \mathrm{O}^{-1}\right)\end{array}$} \\
\hline & 39 DAS & 67 DAS & 39 DAS & 67 DAS & 39 DAS & 67 DAS \\
\hline $\begin{array}{l}\text { P. vulgaris } \\
\text { G. } \max \end{array}$ & $\begin{array}{l}0,574 \mathrm{bc} \mathrm{B} \\
0,543 \text { с B }\end{array}$ & $\begin{array}{l}0,954 \text { a } \mathrm{A} \\
0,864 \text { a A }\end{array}$ & $\begin{array}{l}5,650 \text { a } \mathrm{A} \\
5,650 \text { a } \mathrm{A}\end{array}$ & $\begin{array}{l}5,192 \text { b B } \\
5,242 \text { b B }\end{array}$ & $\begin{array}{l}2,103 \text { с A } \\
2,208 \text { bc B }\end{array}$ & $\begin{array}{l}1,686 \mathrm{c} \mathrm{B} \\
2,447 \mathrm{ab} \mathrm{A}\end{array}$ \\
\hline E. heterophylla (resistente*) & 0,740 a $\mathrm{B}$ & 1,117 a $\mathrm{A}$ & 6,108 a $\mathrm{A}$ & $5,300 \mathrm{ab} \mathrm{B}$ & $2,700 \mathrm{ab} A$ & 2,717 a $\mathrm{A}$ \\
\hline E. heterophylla (suscetível*) & $0,675 \mathrm{ab} B$ & 1,138 a $\mathrm{A}$ & 6,208 a $\mathrm{A}$ & 5,516 a B & 2,825 a $\mathrm{A}$ & $2,642 \mathrm{ab} A$ \\
\hline B. pilosa & 0,554 с B & 1,048 a $\mathrm{A}$ & 5,925 a $\mathrm{A}$ & $5,450 \mathrm{ab} \mathrm{B}$ & 2,368 abc A & $2,332 \mathrm{ab} A$ \\
\hline D. tortuosum & 0,509 c B & 1,004 a $\mathrm{A}$ & 5,900 a $\mathrm{A}$ & $5,325 \mathrm{ab} \mathrm{B}$ & $1,518 \mathrm{~d} \mathrm{~B}$ & $2,158 \mathrm{bc} \mathrm{A}$ \\
\hline
\end{tabular}

* Biótipo resistente e suscetível aos herbicidas inibidores da ALS.

Médias seguidas de mesma letra, minúscula na coluna e maiúscula na linha, não diferem estatisticamente entre si pelo teste de Tukey a $5 \%$ de probabilidade. 
exceção das plantas de $D$. tortuosum. Entre as culturas, a soja demonstrou ser a espécie com maior potencial de utilização no controle cultural das plantas daninhas, porém as plantas de feijão, principalmente devido ao rápido acúmulo inicial de biomassa, podem ser manejadas de forma a ocupar rapidamente os espaços, suprimindo as plantas daninhas que possam aparecer posteriormente. Entre as espécies de plantas daninhas avaliadas, D. tortuosum pode ser considerada a mais suscetivel ao controle cultural, em razão do seu baixo desempenho ecofisiológico.

\section{AGRADECIMENTOS}

Ao CNPq, pelo apoio financeiro.

\section{LITERATURA CITADA}

BEGNA, S. H. et al. Morphology and yield response to weed pressure by corn hybrids differing in canopy architecture. Europ. J. Agron., v. 14, p. 293-302, 2001.

BLANCO, H. G. et al. Observações sobre o período em que as plantas daninhas competem com a soja (Glycine $\max (\mathrm{L}$.) Merr.). O Biológico, v. 39, p. 31-35, 1973.

COBLE, H. D.; WILLIANS, F. M.; RITLE, R. L. Common ragweed (Ambrosia artemisiifolia) interference in soybeans (Glycine max). Weed Sci., v. 29, p. 339-342, 1981.

DOMÍNGUEZ, C.; TENA, M.; DE PRADO, R. Photosynthetic activity, growth and productivity in a striazine-resistant biotype of Solanum nigrum. Plant Physiol. Biochem., v. 32, p. 627-632, 1994.

KREMER, E.; KROPFF, M. J. Comparative growth of triazine-susceptible and -resistant biotypes of Solanum nigrum at different light levels. Ann. Botany, v. 83, p. 637644, 1999.
MASABNI, J. G.; ZANDSTRA, B. H. Physiological characteristics of linuron-resistant Portulaca oleracea. Weed Sci., v. 48, p. 420-425, 2000.

MASCARENHAS, M. H. T. Competição de plantas daninhas com as culturas. Inf. Agropec., v. 8, p. 26-32, 1982.

NOBEL, P. S. Physicochemical and environmental plant physiology. San Diego: Academic Press, 1991. 635 p.

SANTOS, J. B.; SILVA, A. A. Resistência de genótipos de Euphorbia heterophylla L. a herbicidas inibidores da enzima acetolactato sintase (ALS). Viçosa: Universidade Federal de Viçosa, 2000. 37 p. (Relatório. PIBIC/CNPq).

SHARPLES, C. R.; HULL, M. R.; COBB, A. H. Growth and photosynthetic characteristics of two biotypes of the weed black-grass (Alopecurus myosuroides Huds.) resistant and susceptible to the herbicice chlorotoluron. Ann.

Botany, v. 79, p. 455-461, 1997.

STERLING, T. M.; LOWNDS. N. K.; MURRAY. L. W. Picloran-resistant and - susceptible yellow starthristle accessions have similar competitive ability. Weed Sci., v. 49 , p. $42-47,2001$.

VARGAS, L. et al. Resistência de plantas daninhas a herbicidas. Viçosa, MG: Edição dos Autores, 1999. 131 p.

VIDAL, R. A.; TREZZI, M. M. Análise de crescimento de biótipos de leiteira (Euphorbia heterophylla) resistentes e susceptível aos herbicidas inibidores da ALS. Planta Daninha, v. 18, p. 427-433, 2000.

VIEIRA, C. Período crítico de competição entre ervas daninhas e a cultura do feijão (Phaseolus vulgaris L.). R. Ceres, v. 17, p. 354-367, 1970. 